\title{
PARÂMETROS CARDIOPULMONARES E HEMOGASOMÉTRICOS EM OVINOS SOB ANESTESIA INALATÓRIA DURANTE ALTERNÂNCIAS DE DECÚBITO
}

\author{
CARDIOPULMONARY AND BLOOD GAS PARAMETERS IN SHEEP \\ UNDER INHALATIONAL ANESTHESIA DURING DECUBITUS \\ ALTERNATIONS
}

\author{
Liana Villela Gouvea ${ }^{1}$ \\ João Gabriel César Palermo ${ }^{1}$ \\ José Renato Junqueira Borges ${ }^{1}$ \\ Ricardo Miyasaka Almeida ${ }^{1}$ \\ Fábio Henrique Bezerra Ximenes ${ }^{1}$ \\ Roberta Ferro de Godoy ${ }^{1^{*}}$ \\ 1 Universidade de Brasília, Brasília, DF, Brasil. \\ *Autora para correspondência - rdegodoy@rvc.ac.uk
}

\begin{abstract}
Resumo
Os rebanhos de ovinos do Centro-Oeste do Brasil, principalmente da raça Santa Inês, aumentaram muito nos últimos anos. Dessa forma, a casuística clínica também cresceu e a realização de cirurgias de grande porte sem dor e com segurança se faz presente, associada ao uso da anestesia geral inalatória. Este trabalho objetivou avaliar os parâmetros cardiopulmonares e hemogasométricos em ovinos sob anestesia inalatória com ventilação controlada, bem como avaliar a influência de alternâncias de decúbito nos mesmos parâmetros. Os animais foram sedados com acepromazina, induzidos com propofol, intubados e colocados sob anestesia inalatória com isoflurano durante manutenção anestésica. As frequências cardíaca e respiratória, pressão arterial e a concentração de gases sanguíneos foram aferidas a cada cinco minutos por um total de 45 minutos, durante os quais os animais foram alternados entre decúbito lateral direito, dorsal e esquerdo. Os parâmetros aferidos não apresentaram diferenças significativas entre os tempos e decúbitos, concluindo-se que a anestesia geral inalatória com isoflurano mostra-se viável e segura na espécie ovina, pois mantém os parâmetros cardiopulmonares e hemogasométricos em níveis seguros.
\end{abstract}

Palavras-chave: cirurgia; decúbito; hemogasometria.

\begin{abstract}
Sheep herds, especially Santa Ines breed, have grown in the Brazilian Midwest in recent years. Therefore, clinical cases have also grown, and along came the need of conducting major surgeries without pain associated with the use of inhalational anesthesia. This study evaluated the cardiopulmonary and blood gas parameters in sheep under inhalational anesthesia with controlled ventilation, and assessed the effect of decubitus alternations on the parameters. The animals were anesthetized with acepromazine and propofol, intubated and placed on inhalational anesthesia with isoflurane. The respiratory rates, blood pressure and blood gas concentrations were measured every for minutes for a total of 45 minutes during which the animals were alternated right, dorsal and left decubitus. The measured parameters showed no significant differences among the times and positions, concluding that inhalational anesthesia with isoflurane is valid and safe for sheep, as it keeps the cardiopulmonary parameters and arterial blood gases at safe levels.
\end{abstract}


Keywords: blood gas; decubitus; surgery.

Enviado em: 21 abril 2011

Aceito em 15 março 2016

\section{Introdução}

Os rebanhos de ovinos do Centro-Oeste, principalmente os da raça Santa Inês, assim como o número de propriedades, têm aumentado nos últimos anos devido à crescente demanda por carne ovina nessa região. Com isso, a casuística de tratamentos de ovinos no hospital veterinário também aumentou, sendo, em muitos casos, necessária intervenção cirúrgica. Técnicas anestésicas locais são bastante utilizadas em ruminantes em substituição ou em associação com técnicas intravenosas. Isso devese ao fato de estas técnicas oferecerem maior segurança cardiorrespiratória e terem um custo mais baixo $^{(1)}$. Entretanto, para a realização de cirurgias de grande porte sem dor e com segurança, faz-se necessário o uso de anestesia geral inalatória ${ }^{(2,3)}$.

Foi demonstrado que não há diferenças na anestesia com sevoflurano ou desflurano nos achados clínicos cardiopulmonares, hematológicos e bioquímicos nos ovinos em respiração espontânea ${ }^{(2)}$. Diminuição significativa da frequência cardíaca, débito cardíaco e pressão arterial média em cordeiros sob anestesia com isoflurano foram observadas em outro estudo ${ }^{(4)}$, e a queda do débito cardíaco e da pressão arterial média foram proporcionais à quantidade do fármaco vaporizado. Embora tenha ocorrido esta diminuição de aporte sanguíneo, houve associadamente um decréscimo do requerimento de oxigênio, não havendo comprometimento de órgãos vitais. Quando o isoflurano foi comparado ao propofol, um anestésico não volátil, em ovinos, verificou-se que o propofol em infusão contínua causa uma menor depressão cardiovascular em ovelhas gestantes, porém não há diferenças entre os dois fármacos no que concerne ao fluxo sanguíneo nas artérias umbilicais ${ }^{(5)}$.

Além da estabilidade cardiopulmonar, a anestesia geral inalatória em ovinos é interessante por proporcionar uma recuperação anestésica rápida, diminuindo o risco de regurgitação, mas o uso de agentes injetáveis associados pode anular esta vantagem ${ }^{(6)}$. Não há diferença entre desflurano, isoflurano e sevoflurano no que concerne aos efeitos cardiopulmonares e no tempo de recuperação pós-anestésica em ovinos. Os três anestésicos foram considerados viáveis para o uso nesta espécie, embora causem uma pequena depressão cardiopulmonar ${ }^{(6)}$.

A hemogasometria, com as determinações de pressão de oxigênio e gás carbônico e do equilíbrio ácido-básico, representado pelo $\mathrm{pH}$, é usada extensivamente como um indicador da eficiência da troca pulmonar de gases e do status respiratório. Seu uso durante a anestesia geral é recomendado com o objetivo de reduzir a ocorrência de emergências anestésicas ${ }^{(7)}$. Adicionalmente, é utilizada para avaliação de novos anestésicos e procedimentos de ventilação durante a anestesia( ${ }^{(8)}$.

A literatura fornece poucos dados relacionados às alterações que o isoflurano produz sobre os parâmetros hemogasométricos dos ovinos submetidos ao agente e, em especial, às possíveis diferenças que podem ocorrer quando estes animais são submetidos à alterações de decúbito. Desta maneira, o conhecimento de dados relativos ao isoflurano sobre esses parâmetros constitui-se de grande importância para a execução de uma anestesia segura.

Este trabalho objetivou avaliar os parâmetros cardiopulmonares e hemogasométricos em ovinos sob anestesia inalatória com ventilação controlada, bem como avaliar a influência de alternâncias de decúbito nos mesmos parâmetros.

\section{Materiais e Métodos}

Este trabalho foi aprovado pelo Comitê de Ética no Uso Animal (CEUA) da UnB, sob o protocolo no $76850 / 2007$.

Foram utilizadas sete fêmeas, adultas, da raça Santa-Inês, com peso médio de $40 \mathrm{~kg}$. Os ovinos foram mantidos em um piquete experimental com pastagem Tifton, água e sal ad libitum durante todo o período experimental. Os animais foram submetidos ao jejum alimentar e hídrico por 18 e 
12 horas, respectivamente. No dia do procedimento, foram submetidos à pré-anestesia e indução anestésica com acepromazina1\% (Acepran 1\%, Univet S/A, São Paulo, Brasil), na dose de 0,1 mg/kg, e propofol (Propovan, Cristália, Itapira, Brasil), na dose de $4,4 \mathrm{mg} / \mathrm{kg}$, ambos por via intravenosa. Os animais foram entubados, com auxílio de um laringoscópio, com a sonda orotraqueal adaptada com um prolongamento e auxilio de um guia metálico (Figura 1).

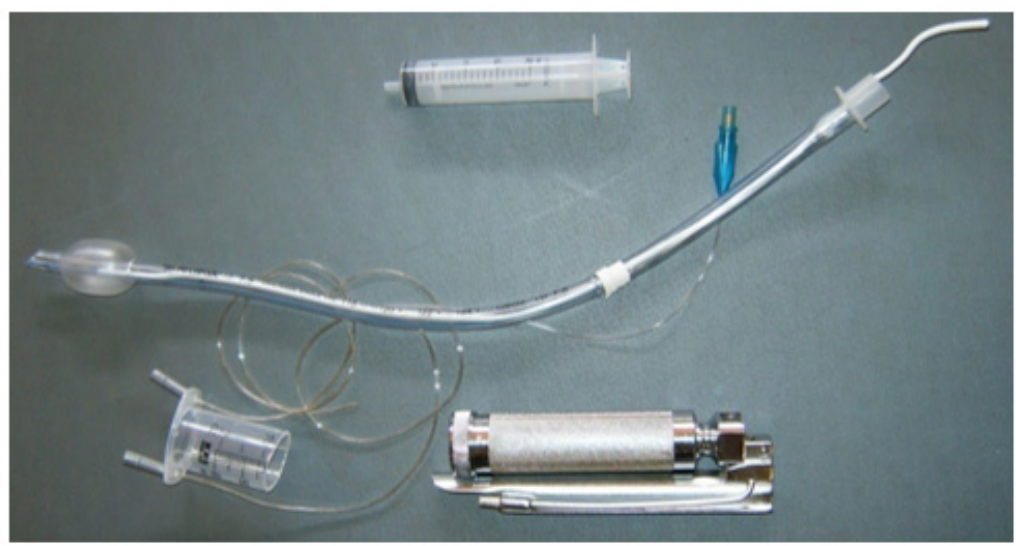

Figura 1: Aparato necessário para intubação de pequenos ruminantes. Seringa para inflar balonete, sonda orotraqueal e guia metálico, laringoscópio e abre-boca feito a partir de uma seringa de $60 \mathrm{~mL}$.

O isoflurano (Isoforine, Cristália, Itapira, Brasil) foi vaporizado, em um vaporizador universal, de maneira controlada para que o animal se mantivesse em plano anestésico cirúrgico. A verificação do plano anestésico se deu através da observação dos reflexos protetores, ou seja, ausência de reflexo palpebral e presença de reflexo corneal.

Inicialmente, o animal foi colocado em decúbito lateral direito e a monitorização realizada com monitores cardíacos com medição de $\mathrm{SpO}_{2}$ (Active "ES", Transform Tecnologia de Ponta Ltda., São Paulo, Brasil), frequência cardíaca e ECG (CardioPET, R\&D Mediq Ltda., São Paulo, Brasil). Frequência respiratória (FR) e temperatura foram monitoradas (T1). Além disto, a artéria metatarsiana dorsal foi cateterizada com um cateter $22 \mathrm{G}$ e foi aferida a pressão arterial invasiva (PAI), sendo considerada a pressão arterial média (PAM). A cada 05 minutos, partindo do momento T0 (T0, T5, T10, T15, T20, T25, T30, T35 e T40), os parâmetros (FC, FR, spO 2 e PAI) foram anotados em uma planilha e o sangue foi coletado para realização da hemogasometria com seringa heparinizada (BD Preset ${ }^{\circledR} 0,7 \times 25 \mathrm{~mm}-21 \mathrm{G} 1$ "). As amostras foram transportadas até o laboratório para processamento num recipiente térmico com gelo e temperatura em torno de $0-4{ }^{\circ} \mathrm{C}$. A amostra de sangue heparinizado foi analisada em um hemogasômetro (Cobas b 121, Roche) para se obterem os parâmetros referentes à $\mathrm{pH}, \mathrm{pCO}_{2}, \mathrm{pO}_{2}$, concentração de bicarbonato $\left(\mathrm{HCO}_{3}^{-}\right)$e excesso de base $(\mathrm{BE})$.

Durante a anestesia, o animal teve seu decúbito alterado para dorsal em T15 e esquerdo em T30, mantendo-se em cada decúbito por 15 minutos, durante os quais foram realizados aferimentos dos parâmetros cárdio-respiratórios e hemogasométricos. Após a finalização dos procedimentos, o animal foi encaminhado para a sala de recuperação anestésica.

Para análise estatística, utilizou-se o programa EXCELL do Windows e o programa GraphPad InStat. O teste estatístico utilizado para a análise dos dados de monitorização foi o método não-paramétrico e não pareado de Mann-Whitney. Para avaliação dos parâmetros hemogasométricos, foi utilizado o teste não-paramétrico e não pareado de Mann-Whitney.

\section{Resultados e Discussão}

As médias dos parâmetros avaliados foram tabuladas e expressas graficamente (Figura 2). 


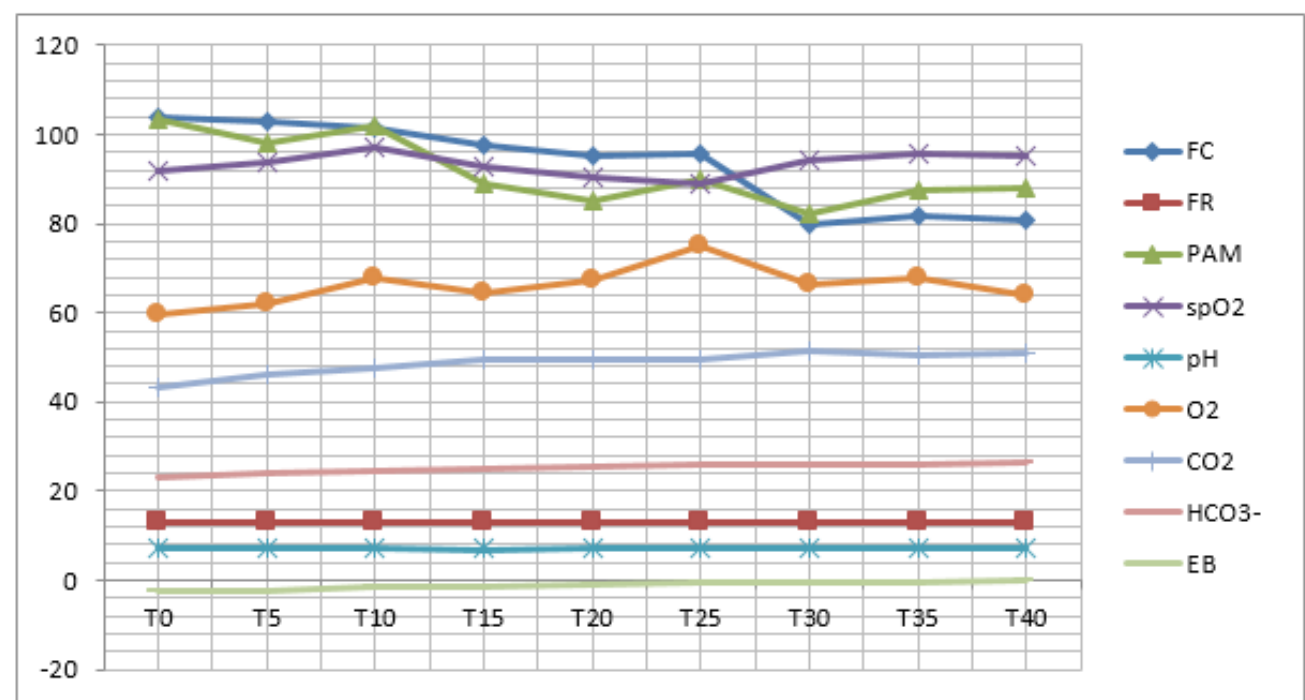

Figura 2: Variação dos diversos parâmetros cardiopulmonares e hemogasométricos em ovinos, nos decúbitos lateral direito (T0-T10), dorsal (T15-T25) e lateral esquerdo (T30-T40).

Os parâmetros cardiopulmonares foram avaliados pelo teste de Mann-Whitney $(p<0,05)$.

A média da frequência cardíaca (FC) dos animais, expressa em batimentos por minuto (bpm), apresentou os seguintes resultados: em decúbito lateral direito, T0-103,8; T5-103 e T10-101,3; em decúbito dorsal, T15-97,7; T20-95 e T25-95,7; em decúbito lateral esquerdo, T30-80; T35-81,5 e T40-80,8. Todos os anestésicos inalatórios causam depressão do sistema cardiovascular dosedependente; contudo, a magnitude dessas alterações pode ser influenciada pelo fármaco empregado ou pela associação com outros agentes ${ }^{(13)}$. Adicionalmente, foi observado que, em ovinos submetidos a 60 minutos de anestesia com 1 e 1,5\% de halotano e isoflurano, respectivamente, a frequência cardíaca apresentou redução em relação ao valor basal, porém sem diferença entre os fármacos ${ }^{(14)}$. No presente experimento, a diferença da FC não foi significativa nos diferentes decúbitos; no entanto, houve diminuição gradual da FC ao longo do tempo de anestesia. A FC em ovinos anestesiados com halotano deve variar de 80 a 100 bpm $^{(10)}$.

A frequência respiratória (FR) média dos ovinos, expressa em movimentos respiratórios por minuto (mpm), manteve-se constante em todos os decúbitos, com o valor de 12,85 mpm. Em ruminantes, a distensão do rúmen pela contínua produção de gases influencia diretamente a ventilação ${ }^{(15)} \mathrm{e}$, associado ao decúbito lateral, há potencialização desses efeitos ${ }^{(16)}$. Entretanto, não foi observada tal alteração nos padrões ventilatórios favorecidos pelo jejum prévio e pelo pouco tempo que esses animais foram submetidos ao decúbito quando comparado aos experimentos anteriores.

A pressão arterial média (PAM), expressa em $\mathrm{mmHg}$, apresentou os seguintes valores: em decúbito lateral direito os valores foram T0-103,3; T5-98,2 e T10-101,7; em decúbito dorsal, T15-89; T20-85 e T25-90; e em decúbito lateral esquerdo, T30-82,3; T35-87,3 e T40-88,2. O isoflurano é o agente que induz maior redução dos valores da pressão arterial, sendo este efeito atribuído à redução na resistência vascular periférica e discreta redução no débito cardíaco ${ }^{(13)}$. No entanto, em outro experimento, foi observado que tanto o halotano, quanto o isoflurano e o sevoflurano não promoveram hipotensão arterial em ovinos, merecendo ênfase o grupo de animais tratados com halotano, pois, mesmo sem diferença estatística, esse grupo manteve patamares mais elevados que o observado nos demais grupos $^{(9)}$. No presente experimento, também não foi observada hipotensão com o uso do isoflurano alternando decúbitos, mas uma diminuição ao longo do tempo de anestesia.

Os resultados observados na avaliação da $\mathrm{spO}_{2}$, expressa em porcentagem de saturação de oxigênio (\%), em decúbito lateral direito foram T0-92; T5-93,6 e T10-97,3; em decúbito dorsal, T15-92,8; T20-90,3 e T25-89,1; e em decúbito lateral esquerdo, T30-94; T35-95,8 e T40-95.

Em nenhum destes parâmetros estudados foi verificada diferença estatística entre os tempos e decúbitos. Esses resultados corroboram os achados de outros experimentos ${ }^{(2,10)}$ que também comprovaram que a anestesia geral inalatória com isoflurano promove alterações não significativas nos parâmetros cardiorrespiratórios. Porém, estes autores não avaliaram a troca de decúbito durante procedimento 
anestésico.

Os parâmetros hemogasométricos, também avaliados pelo teste de Mann-Whitney $(\mathrm{p}<0,05)$, não apresentaram diferenças significativas entre tempos e decúbitos. $\mathrm{O} \mathrm{pH}$ manteve-se praticamente constante em todos os decúbitos avaliados. Em decúbito lateral direito os valores foram T0-7,34; T5-7,32 e T10-7,32; em decúbito dorsal, T15-7,31; T20-7,32 e T25-7,33; e em decúbito lateral esquerdo, T30-7,32; T35-7,33 e T40-7,33. Em estudo comparando halotano, isoflurano e sevoflurano em cabras, o isoflurano foi o que apresentou níveis de $\mathrm{pH}$ mais altos ${ }^{(17)}$. No presente estudo, não foi observada alteração em relação aos níveis basais com o uso de isoflurando em diferentes decúbitos. A manutenção desse parâmetro em níveis normais é reflexo dos outros parâmetros hemogasométricos que também se mantiveram próximos aos níveis normais.

A média da $\mathrm{pO}_{2}(\mathrm{mmHg})$, pressão parcial de oxigênio, apresentou os seguintes valores em respiração espontânea: em decúbito lateral direito os valores foram T0-59,6; T5-62,0 e T10-67,7; em decúbito dorsal, T15-64,2; T20-67,2 e T25-74,9; e em decúbito lateral esquerdo, T30-66,4; T35-67,8 e T4064,0 . Em um estudo comparativo do sangue arterial, capilar e venoso em ovinos adultos clinicamente saudáveis, e os dados basais de $\mathrm{pO}_{2}$ arterial foram em torno de $1,54 \mathrm{kPa} \approx 86,55 \mathrm{mmHg}$ (em que $1 \mathrm{kPa}$ equivale a $7,5 \mathrm{mmHg})^{(11)}$. Assim sendo, os valores aqui encontrados foram inferiores aos achados, pelo último pesquisador ${ }^{(11)}$, em animais não anestesiados, o que justifica esta diferença.

A média da $\mathrm{pCO}_{2}(\mathrm{mmHg})$, pressão parcial de dióxido de carbono, obteve os seguintes valores: em decúbito lateral direito, T0-43,4; T5-46,3 e T10-47,7; em decúbito dorsal, T15-49,62; T20-49,68 e T25-49,65; e em decúbito lateral esquerdo, T30-51,38; T35-50,3 e T40-50,7. De acordo com um experimento em ovelhas não anestesiadas, os dados basais de $\mathrm{pCO}_{2}$ arterial são em torno de $4,91 \mathrm{kPa}$ $\approx 36,82 \mathrm{mmHg}^{(11)}$. No presente estudo, encontramos valores superiores aos apenas citados. Porém, nossas avaliações foram realizadas em animais anestesiados, o que explica esta diferença.

Os achados de $\mathrm{pO}_{2}$ e $\mathrm{PCO}_{2}$ arteriais deste estudo corroboram o fato da ocorrência de depressão respiratória, consequentemente possível acidose respiratória, ser favorecida pela utilização do isoflurano, embora a depressão seja maior com uso de halotano ${ }^{(17)}$.

A média da concentração de $\mathrm{HCO}_{3}^{-}(\mathrm{mmol} / \mathrm{L})$, bicarbonato de sódio, observada nas diversas posições, apresentou os seguintes valores: em decúbito lateral direito, T0-23,22; T5-23,90 e T10-24,57; em decúbito dorsal, T15-24,81; T20-25,31 e T25-25,80; e em decúbito lateral esquerdo, T30-26,10; T3526,01 e T40-26,28. Parâmetros basais de $\mathrm{HCO}_{3}^{-}(\mathrm{mmol} / \mathrm{L})$, em ovelhas não anestesiadas são em torno de $20,43 \mathrm{mmol} / \mathrm{L}^{(11)}$. No presente estudo, foram observados níveis ligeiramente maiores, que podem ser explicados pelo efeito da anestesia no balanço áciso-base. A medição do $\mathrm{HCO}_{3}^{-}$no sangue é da maior importância na clínica, porque indica a capacidade de o organismo manejar quantidades adicionais de ácidos orgânicos, além de servir como base de cálculo para o déficit de base (DB) ${ }^{(9)}$. $\mathrm{O}$ aumento dos níveis observados neste estudo é considerado como uma medida compensatória ao aumento da $\mathrm{pCO}_{2}$ arterial observado anteriormente.

A média do EB (excesso de base - $\mathrm{mmol} / \mathrm{L}$ ) apresentou os seguintes valores: no decúbito lateral direito, T0-2,45; T5-2,17 e T10-1,58; em decúbito dorsal: T15-1,62; T20-1,04 e T25-0,57; e em decúbito lateral esquerdo, T30-0,47; T35-0,35 e T40-0,11. Os resultados obtidos condizem com os valores encontrados em outros estudos dos gases sanguíneos de ovinos ${ }^{(8,12)}$. Porém estes estudos apenas citados não abordaram a problemática da alternância de decúbito.

Neste experimento com anestesia geral inalatória em ovinos com isoflurano não foram observadas diferenças significativas $(\mathrm{p}<0,05)$, tanto nos parâmetros cardiopulmonares quanto nos hemogasométricos em relação aos três decúbitos analisados (Figura 2), embora os sinais de depressão cardiorrespiratória tenham sido observados ao longo do tempo de anestesia.

\section{Conclusões}

A anestesia geral inalatória com isoflurano mostra-se viável e segura na espécie ovina, pois mantém os parâmetros cardiopulmonares e hemogasométricos em níveis seguros. Além disso, pode-se inferir que a mudança de decúbito também não interfere na qualidade do procedimento anestésico, pois os parâmetros analisados não sofreram alterações significativas quando da alternância de decúbito. Esse fato se mostra de fundamental importância para a realização de procedimentos cirúrgicos mais 
complexos ou de emergência que necessitem tais alterações posturais.

\section{Agradecimentos}

Os autores agradecem ao Decanato de Pesquisa e Pós-Graduação e à FINATEC pelo apoio financeiro. E à Dra. Júlia de Miranda Moraes pelo auxílio na análise estatística.

\section{Referências}

1. Skarda, RT Local and regional anesthesia in ruminants and swine. Veterinary Clinics of North America: Food Animal Practice. 1996; 12 (3): 579 - 625.

2. Hikasa Y, Hokushin S, Takase K, Ogasawara S. Clinical, cardiopulmonary, hematological and serum biochemical effects of sevoflurane and isoflurane anesthesia in oxygen under spontaneous breathing in sheep. Small Ruminant Research. 2000; 36(3): 241-249.

3. Voss LJ, Ludbrook GL, Grant C, Sleigh J, Barnard JPM. Cerebral cortical effects of desflurane in sheep: comparison with isoflurane, sevoflurane and enflurane. Acta Anaesthesiol Scand. 2006; 50(3): 313-319.

4. Brett CM, Teidel DF, Heymann MA, Rudolph AM. The cardiovascular effects of isoflurane in lambs. Anesthesiology. 1987; 67(1): 60-65.

5. Gaynor JS, Wertz EM, Alvis M, Turner AS. A comparison of the haemodynamic effects of propofol and isoflurane in pregnant ewes. Journal of Veterinary Pharmacology and Therapeutics. 1998; 21(1): 69-73.

6. Mohamadnia AR, Hughes G, Clarke KW. Maintenance of anesthesia in sheep with isoflurane, desflurane or sevoflurane. Veterinary Records. 2008; 163(7): 210-215.

7. Grubb, TL, Muir WW. Anaesthetic emergencies and complications - part 1. Equine Vet. Educ., 1998; 10(2): 98-109.

8. Gouvêa LV. Análise hemogasométrica arterial e venosa de ovinos submetidos à transposição carotídea e indução de desequilíbrios ácido-básicos. Brasília: Faculdade de Agronomia e Medicina Veterinária, Universidade de Brasília, 2009, 49p. Dissertação de Mestrado. http://bdtd.bce.unb.br/tedesimplificado/tde busca/arquivo.php? codArquivo $=5042$

9. Junior EM. Anestesia inalatória em ovinos: estudo comparativo entre o halotano, isoflurano e sevoflurano. Tese de Doutorado - Universidade de São Paulo. Faculdade de Medicina Veterinária e Zootecnia. Departamento de Cirurgia. São Paulo. 2012. http://www.teses.usp.br/teses/disponiveis/10/10137/tde27092012-174918/publico/EWALDO_MATTOS_JUNIOR.pdf

10. Natalini CC, Mollerke RO, Motta U, Teixeira AS, Endler JO, Jobim GB, Jobim GO. Anestesia geral em ovinos submetidos a cirurgia fetal experimental. Acta Cirúrgica Brasileira. 1993; 8: 63-67.

11. Sobiech P, Stopyra A, Kuleta Z, Zbanyszek M, Milewski S. Acid-base balance parameters of arterial, venous and capillary blood in sheep. Bull Vet Inst Pulawy . 2005; 49: 125-127.

12. Leal MLR, Soares PC, Bertagnon HG, Silva PEG, Ortolani EL, Benesi FJ. Efeito da refrigeração sobre o exame hemogasométrico em sangue venoso de ovinos. Brazilian Journal of Veterinary Research and Animal Science. 2006;43: 80-85.

13. Steffey EP, Mama KR. Inhalation anesthetics. In: Tranquilli WJ, Thurmon JC, Grimm KA, editors. Lumb \& Jones' Veterinary Anesthesia and Analgesia. Ames, Iowa, USA: Blackwell; 2007. pp. 355-395.

14. Gençcelep M, Atasoy N, Tas A. The effects of inhalation anaesthetics (halothane and isoflurane) on certain 
clinical and haematological parameters of sheep. Small Ruminant Research. 2004; 53:157-160.

15. Ungerer T, Orr JA, Bisgard GE, Will JA. Cardiopulmonary effects of mechanical distension of the rumen in nonanesthetized sheep. American Journal of Veterinary Research. 1976; 37:807-10.

16. Fujimoto JI, Lenehan TM. The influence of body position on the blood gas and acid-base status of halothaneanesthetized sheep. Veterinary Surgery. 1985; 14: 169-172.

17. Hikasa Y, Okuyama K, Kakuta T, Takase K, Ogasawara S. Anesthetic potency and cardiopulmonary effects of sevoflurane in goats: comparison with isoflurane and halothane. Canadian Journal of Veterinary Research. 1998; 62:299-306. 